\title{
Kurze Zündschnur: Ärger - Wut - Aggression
}

\section{Ambulantes psychotherapeutisches Gruppenkonzept für Männer mit aggressivem Verhalten}

\author{
Romy Schröter ${ }^{1} \cdot$ Martin Lambert $^{1} \cdot$ Anne Karow $^{1} \cdot$ Daniel Lüdecke ${ }^{1} \cdot$ Jürgen Gallinat ${ }^{1} \cdot$ Peer Briken $^{2}$
}

Eingegangen: 4. Dezember 2020 / Angenommen: 19. Februar 2021 / Online publiziert: 18. März 2021

(c) Der/die Autor(en) 2021

\section{Zusammenfassung}

Verschiedene psychische Erkrankungen, wie Persönlichkeitsstörungen, sind mit einem Risiko für gewalttätiges Verhalten assoziiert. Dieses Risiko wird jedoch nur selten durch gezielte psychotherapeutische Interventionen adressiert. Vielmehr werden im psychiatrischen Setting aggressive und impulsive Patienten oftmals isoliert und auf geschützten Stationen aufgenommen. Dies kann beispielsweise durch Stigmatisierung und das Schüren von Ängsten aggressives Verhalten auch begünstigen. Aufgrund von aggressivem Verhalten kommt es zudem häufig zu kurzfristigen disziplinarischen Entlassungen oder konflikthaften Eskalationen im Stationsalltag. Insgesamt besteht sowohl im stationären als auch im ambulanten Setting eine Versorgungslücke. Es mangelt an spezifischen psychotherapeutischen Angeboten, die Patienten Strategien zur Bewältigung von aggressivem Verhalten vermitteln können.

Ein Patientenkollektiv, an dem diese Versorgungslücke deutlich wird, sind Männer mit Persönlichkeitsstörungen. Speziell für diese Patienten wurde ein gruppenpsychotherapeutisches Konzept entwickelt, welches hier vorgestellt werden soll: Die Ärger-Wut-Aggressions(AeWA)-Gruppe. Das Konzept nimmt männliche Patienten mit Störung der Impulskontrolle in den Blick. Dabei wird auf emotionale und entwicklungspsychologische Kernbedürfnisse eingegangen und eine Vielzahl an kognitiven, behavioralen und emotionsfokussierten Techniken vermittelt. Diese sollen eine funktionale Emotionsregulation fördern und gleichzeitig das Auftreten von aggressiven Verhaltensweisen und das damit verbundene Risiko für Straffälligkeit verringern.

Schlüsselwörter Versorgungslücke · Persönlichkeitsstörung · Impulsivität · Gewalt · Emotionsregulation · Gruppentherapie

Romy Schröter, M.Sc.

r.schroeter@uke.de

1 Zentrum für Psychosoziale Medizin, Klinik und Poliklinik für Psychiatrie und Psychotherapie, Universitätsklinikum Hamburg-Eppendorf, Martinistraße 52, 20251 Hamburg, Deutschland
2 Zentrum für Psychosoziale Medizin, Institut für Sexualforschung, Sexualmedizin und Forensische Psychotherapie, Universitätsklinikum Hamburg-Eppendorf, Hamburg, Deutschland 


\title{
Short fuse: anger-rage-aggression
}

Outpatient psychotherapeutic group concept for men with aggressive behavior

\begin{abstract}
Various mental illnesses, such as personality disorders, are associated with a risk of violent behavior; however, this risk is rarely addressed by specific psychotherapeutic interventions. Instead, in the psychiatric setting aggressive and impulsive patients are often isolated and admitted to closed wards. This can even promote aggressive behavior, e.g. by stigmatization and stirring up fears. In addition, aggressive behavior often leads to short-term disciplinary dismissals or conflictual escalations in everyday ward life. All in all, there is a gap in mental healthcare in both the inpatient and outpatient setting. There is a lack of specific psychotherapeutic services that help patients to improve strategies for coping with aggressive behavior.

One group of patients where this gap in care is evident are men with personality disorders. A group psychotherapeutic concept has been developed especially for these patients, which is presented here: the anger-rage-aggression group (AeWA). The concept focuses on male patients with impulse control disorders. It addresses core emotional and developmental psychological needs and teaches a variety of cognitive, behavioral and emotion-focused techniques. These are designed to promote functional emotion regulation and thus also to reduce the occurrence of aggressive behavior with its associated risk for delinquency.
\end{abstract}

Keywords Healthcare gap $\cdot$ Personality disorder $\cdot$ Impulsivity $\cdot$ Violence $\cdot$ Emotion regulation $\cdot$ Group therapy

\section{Hintergrund und aktuelle Situation}

Aggressionen und Gewalt zählen zu den grundlegenden, täglichen und ältesten Problemen in psychiatrischen Institutionen. Im Zusammenhang damit stehen häufig psychische Störungen wie Substanzmissbrauch, psychotische Erkrankungen und schwere Persönlichkeitsstörungen (Herpertz et al. 2016; Mancke et al. 2018; Azevedo et al. 2020). Grenzüberschreitendes Verhalten der Patienten stellt in der Allgemeinpsychiatrie im ambulanten und im stationären Rahmen eine alltägliche Herausforderung für das Behandlungsteam dar: Fehlende Krankheitseinsicht, rigide Charakterstrukturen und geringe Flexibilität für die Umstellung der problematischen Verhaltensweisen erschweren das therapeutische Arbeiten. Vor allem in Akutsituationen kommt es zu interpersonellen Konflikten. Diese Konflikte zeichnen sich insbesondere dadurch aus, dass Patienten eine fordernde Grundhaltung gegenüber dem therapeutischen Team zeigen, welche Suiziddrohungen, das Bestehen auf sofortiger Aufnahme und Notfallmedikation beinhalten kann. Noch schwieriger werden diese Situationen, wenn die Patienten in ihren Anliegen inkonsistent sind: So kann nach akuter Aufnahme kurze Zeit später wieder auf der sofortigen Entlassung bestanden werden. Im besonderen Maße geraten Behandler durch verbal oder physisch aggressives Verhalten bis hin zur Gewalttätigkeit in Bedrängnis (May and Grubbs 2002; Steinert and Traub 2016). Diese Verhaltensweisen erfordern eine unmittelbare Reaktion vonseiten der Behandler. In der Praxis mangelt es jedoch häufig an psychotherapeutischen Angeboten, um mit den beschriebenen Verhaltensweisen adäquat umzugehen.
In der Akutsituation kommt es zudem häufig zum Einsatz restriktiver Maßnahmen wie Fixierung, Sedierung und Schutzisolation, welche wiederum hohe Kosten im Gesundheitssystem verursachen und gleichzeitig nur eine kurzfristige Lösung darstellen (Rubio-Valera et al. 2015). Weiterhin sind konflikthafte Eskalationen im Stationsalltag und disziplinarische Entlassungen keine Seltenheit. Dies führt bei Patienten mit aggressiver Symptomatik zu einem hohen Leidensdruck und beim Bewusstwerden der eigenen Verhaltensweisen zu Selbststigmatisierung (Schandrin et al. 2019). Diese restriktiven Formen sind keine nachhaltigen Methoden, um die dysfunktionalen Verhaltensweisen langfristig zu bessern. Im Gegenteil: Oftmals werden Patienten mit gewalttätigen und impulsiven Verhaltensdurchbrüchen im psychiatrischen Setting isoliert und lediglich auf geschützten Stationen behandelt. Dies kann Stigmatisierung fördern, Ängste schüren und weiteres aggressives Verhalten begünstigen (Cowman et al. 2017).

Das kognitiv ausgerichtete Behandlungsprogramm „Reasoning und Rehabilitation“ (R\&R; Ross et al. 1988) wird als einer der am häufigsten angewendeten Ansätze zur Straftäterbehandlung beschrieben (Antonowicz 2005). Ein weiteres Konzept ist die Präventionsambulanz in Ansbach (Nitschke et al. 2020), mit dem Ziel, psychisch Kranke mit Risikoprofil davor zu bewahren, Straftaten zu begehen, die eine längere Behandlung im Maßregelvollzug zur Folge hätte. Weiterhin gibt es forensische Ambulanzen, die auch präforensischen Patienten und deren Angehörigen Anlaufstellen bieten. Diese Angebote stellen unverzichtbare Stützen für die erfolgreiche Reintegration der Patienten dar. 
Der stationäre Bereich ist zumeist maximal ausgelastet (Klauber et al. 2018), und spezialisierte stationäre Settings, beispielsweise mit dem Schwerpunkt Dialektisch-Behaviorale Therapie (DBT; (Linehan 2008), sind überwiegend für weibliche Patientinnen mit Borderline-Persönlichkeitsstörung ausgelegt. Gerade für männliche Patienten fällt es den behandelnden Teams oft schwer, ein geschütztes therapeutisches Setting zu finden, in welchem der Fokus auf aggressivem Verhalten liegt und dieses offen thematisiert werden kann. Auch im ambulanten Setting gestaltet sich die Suche nach entsprechend ausgerichteten Einzeltherapieplätzen schwierig, und diese sind zudem mit langen Wartezeiten verknüpft. Primär präventive Gruppenangebote, wie die des Gewaltpräventionszentrums VAP e. V.; http://www. gewaltpraeventionszentrum.de), richten sich eher an Bildungseinrichtungen oder an die mit Aggression konfrontierten Berufsgruppen. Unstrukturierte verhaltenstherapeutische Therapiekonzepte zeigen selten eine nachhaltige Wirkung (Fiedler and Herpertz 2016). Psychodynamische Therapiekonzepte wie Mentalisierungsbasierte Therapie (MBT) (Bateman und Fonagy 2006) sowie strukturierte verhaltenstherapeutische Therapiekonzepte wie Dialektisch-Behaviorale Therapie (Linehan 2008) oder Schematherapie (Young 1990) nehmen eine zentrale Rolle ein.

Bis heute gibt es in Allgemeinpsychiatrien und im ambulanten Bereich nur unzureichende Behandlungsangebote für Patienten mit gewalttätigen Impulsen bei Persönlichkeitsstörungen - insbesondere, wenn es sich um männliche Patienten handelt.

Den Behandlern fehlen nachhaltige therapeutische Konzepte in ambulanter und stationärer Behandlung, mit deren Hilfe Patienten Techniken erlernen können, das eigene aggressive Verhalten zu verstehen und zu ändern. Dabei spielt die Auflösung der Fremd- und Selbststigmatisierung eine zentrale Rolle (Aydin and Fritsch 2015). Für Patienten ist es notwendig, ein konstantes, geschütztes therapeutisches Setting zu schaffen, in dem auch soziale Kompetenzen entwickelt werden können. Die sich hieraus ergebende Behandlungsnotwendigkeit steht allerdings der von Therapeuten häufig vertretenen Ansicht gegenüber, dass Menschen mit einer Persönlichkeitsstörung nicht lernfähig und daher auch nicht therapiefähig seien (Gendreau et al. 2004). Hinsichtlich der Versorgungssituation ist von einer psychotherapeutischen Lücke für Patienten mit schweren psychischen Erkrankungen, die aggressive Verhaltensweisen aufzeigen, auszugehen. Bleiben diese Patienten unbehandelt, droht u. U. eine Forensifizierung, d.h. es besteht die Gefahr, dass die Patienten früher oder später mit dem Gesetz in Konflikt kommen. Im Jahr 2019 wurden in Deutschland 92.742 Personen aufgrund von Gewaltverbrechen (Körperverletzung, Totschlag, Mord) verurteilt - hiervon waren 89,6\% männlich (Statistisches Bundesamt 2020).
Ein beispielhafter Ausweg ist die Etablierung eines strukturierten, psychoedukativ und psychotherapeutisch ausgerichteten ambulanten Gruppenangebots für diese spezifische Patientenklientel. Dies wird am Universitätsklinikum Hamburg-Eppendorf seit 2018 in der Ärger-WutAggression-Gruppe (AeWA-Gruppe) umgesetzt.

In den letzten Jahren haben störungsspezifisch ausgerichtete manualisierte Gruppenpsychotherapien auch in der Forschung an Bedeutung gewonnen (Burlingame et al. 2012) und zeichnen sich u. a. durch die gute Überprüfbarkeit aus. Für Störungsbilder wie Angst- und Zwangsstörungen, Depressionen, Schizophrenie, Abhängigkeitsstörungen und Essstörungen existieren bereits wissenschaftlich untersuchte Angebote (Hautzinger 2011; Schaub 2018; Sipos und Schweiger 2018). Für psychisch erkrankte Patienten mit gewalttätigen Verhaltensweisen gibt es im Hinblick auf die Wirksamkeit strukturierter (Gruppen-)Therapien im allgemein-psychiatrischen Bereich allerdings momentan keine empirisch belastbaren Studien (Steinert und Hirsch 2020), sodass es dringend weiterer Forschung bedarf. Insgesamt findet das Format Gruppentherapie derzeit noch zu wenig Beachtung (Bundespsychotherapeutenkammer 2018), obwohl die Wirksamkeit von Gruppentherapie vielfach nachgewiesen werden konnte (Feng et al. 2012; Polnay et al. 2014) und so therapeutische Ressourcen sinnvoll gebündelt werden könnten.

\section{Die Ärger-Wut-Aggression-Gruppe}

In einem Pilotprojekt über 2 Jahre wurde ein gruppenbasiertes Emotionsregulationstraining für Männer mit gewalttätigen Verhaltensweisen erprobt und hinsichtlich der Anwendbarkeit im klinischen Alltag geprüft. In einer zukünftigen Studie soll diese strukturierte Intervention durchgeführt und hinsichtlich ihrer Wirksamkeit überprüft werden. Im Rahmen des Pilotprojekts wurde das therapeutische Konzept der Gruppe fortlaufend an den Unterstützungsbedarf der Teilnehmer angepasst. Hierbei wurde deutlich, dass neben der Teilnahme an der Gruppentherapie ein zusätzlicher Bedarf an therapeutischen Einzelgesprächen bzw. Paar- und Familiengesprächen bestand. Diese Erfahrungen sollen in dem Gruppentherapiekonzept berücksichtigt werden.

Mit der AeWA-Gruppe wurde ein neues psychotherapeutisches Konzept für Männer mit Störungen der Impulskontrolle entwickelt. Dabei wird auf emotionale und entwicklungspsychologische Kernbedürfnisse, wie z.B. Bindung, fokussiert und eine Vielzahl an kognitiven, behavioralen und emotionsfokussierten Techniken vermittelt. Diese sollen eine funktionale Emotionsregulation fördern und damit auch das Auftreten von gewalttätigen Verhaltensweisen verringern. 
Im Rahmen des vorliegenden Artikels möchten wir, basierend auf unseren therapeutischen Erfahrungen mit der genannten Klientel, ein personenzentriertes Modell, inklusive ausführlicher Deliktanalyse, und dessen Implikationen für die Behandlung präsentieren. In der praktischen Umsetzung bedeutet dies ein psychotherapeutisch geleitetes Gruppenangebot im ambulanten Setting: die AeWA-Gruppe.

Sie ist dabei auf lerntheoretischen Prinzipien aufgebaut und basiert auf kognitiv-verhaltenstherapeutischen Methoden, die darauf abzielen, sozial angemesseneres Verhalten aufzubauen und impulsives wie aggressives Verhalten zu verringern. Der Aufbau ist modular und umfasst 3 Einzeltherapieeinheiten, 20 Gruppentherapieeinheiten sowie bei Bedarf 2 Eltern-/Paartherapieeinheiten. Im Einzeltherapie werden v. a. Wahrnehmungsverzerrungen thematisiert und ein konstruktiver Umgang mit Konflikten eingeübt.

Die Indikation wird anhand einer Testdiagnostik mit dem diagnostischen Kurzinterview bei psychischen Störungen (M.I.N.I. Interview; Sheehan et al. 1998) gestellt. Persönlichkeitsstörungen werden z.B. durch den PID5 BF (Krueger et al. 2012) erhoben. Darüber hinaus wird die aktuelle Gewalttätigkeit zum Zeitpunkt der Aufnahme in die Gruppe sowie im Behandlungszeitraum mittels der Modified Overt Aggression Scale (MOAS; Kay et al. 1988) erhoben. Neben der aktuellen und anamnestischen Gewalttätigkeit werden die aus der Literatur bekannten Risikofaktoren mittels z. B. HCR-20 (Version 2; Webster et al. 2017) sowie weitere Fragebogen durch eine approbierte Psychotherapeutin ermittelt. Weiterhin werden der Clinical Global Impression Scale (CGI; Guy 1976) und die Global Assessment of Functioning Scale (GAF; American Psychiatric Association 1994) ermittelt. Neben soziografischen Fragen und Fragen zu Vorbehandlungen werden den Patienten sowohl bei Behandlungsende als auch zum Katamnesezeitpunkt nichtstandardisierte Fragebogen zur Beurteilung gegeben. Hierbei handelt es sich um Fragen zur Therapiebeurteilung, zum momentanen Befinden, zu Anschlusstherapien. Vor dem Beginn der Gruppe erfolgen mindestens 3 Einzelgespräche zu Deliktanamnese und -analyse sowie zur Einschätzung des Risikos für Gewaltdelikte. Typische Behandlungsindikationen umfassen häusliche Gewalt, Verurteilungen wegen Körperverletzungen, Verwicklungen in Schlägereien, Aggressivität, Feindseligkeit oder Androhungen von Gewalt. Zudem werden in den Einzelsitzungen psychoedukative Elemente zu Persönlichkeitsstörungen eingesetzt. $\mathrm{Zu}$ Beginn der Gruppentherapie werden v.a. kognitiv-verhaltenstherapeutische Interventionen wiederholt, die auf den Bedarf bestimmter Persönlichkeitsakzentuierung bzw. -störungen zugeschnitten sind. Hinzu kommen Anteile aus klassischen Rückfallpräventionsprogrammen für Patienten mit Suchterkrankungen oder schädlichem Suchtmittelkonsum. Vor dem Hintergrund der strukturellen Störungen zeigen sich häufig komorbide psychische Störungen, welche durch
Tab. 1 Therapeutischer Rahmen, Intervention und Vorgehen in der AeWA-Gruppe

Therapeutischer Rahmen

Verlässliches und begrenztes Beziehungsangebot

Besprochene Regeln gelten auch für Therapeuten (Pünktlichkeit etc.)

Gesprächsfokus auf aktuelle Lebenssituationen (Deliktanamnese

u. -analyse)

Nahe und realistische Ziele individuell formulieren und Hoffnung entwickeln

Offener Gesprächskreis in geschütztem Raum

Inhaltliche Bausteine der Intervention

Definition der Hauptprobleme: Krise, Befinden, bisherige (dysfunktionale) Strategien

Psychoedukation: Vermittlung von Wissen über die Erkrankung und ihre Behandlung

Affektregulation und Strategien zum Umgang mit unangenehmen

Gefühlen

Förderung sozialer Kompetenz

Förderung der Motivation, zukünftig ein deliktfreies Leben führen zu wollen

Rückfallprophylaxe erarbeiten und weitere Behandlungsmöglichkeiten besprechen

Ablösung vom Therapeuten

Vorgehen: spezifische Behandlungsgruppe

Gruppenleiterin: psychologische Psychotherapeutin

Gruppenteilnehmer: bis 12 Patienten

Gruppentherapeutische Gespräche: 20 Sitzungen, einmal in der Woche für $90 \mathrm{~min}$

Einzeltherapeutische Gespräche über den Behandlungszeitraum: 3

Familien-/Paargespräche über den Behandlungszeitraum: 2

Haltung der Leiterin: Vermittlerin von Informationen und Wissen

Stil: empathisch-steuernd, ressourcenorientiert und strukturierend, transparent

adaptierte Konzepte der Dialektisch-Behavioralen Therapie (Linehan 1996) und Schematherapie (Young 1990) adressiert werden. Den therapeutischen Rahmen, Interventionen sowie das Vorgehen in der AeWA-Gruppe fasst Tab. $1 \mathrm{zu}$ sammen.

\section{Grenzen des Gruppenkonzeptes}

Liegt die Diagnose einer antisozialen Persönlichkeitsstörung mit deutlicher Psychopathie vor, z. B. Gefühllosigkeit ohne jegliche Empathie/Gewissensbisse, ist eine Therapie aufgrund mangelnder Veränderungsmotivation im Rahmen des Gruppenkonzepts nicht sinnvoll durchführbar. Sie kann gar eine Verstärkung der Charakterpathologie oder bedrohliche Situationen für den Therapeuten zur Folge haben. Weitere Kontraindikationen für eine Behandlung im Rahmen der AeWA-Gruppe sind sadistisch-gewalttätiges Verhalten in der Vorgeschichte, das zu schwerer Körperverletzung oder Tod geführt hat, reaktive Psychosen, mangelnde Intelligenz sowie fehlende Therapiemotivation.

Die Erfahrung nach nunmehr 2 Jahren des laufenden Konzeptes hat gezeigt, dass in einem rein gruppenbasierten 
Konzept für den Patienten durchaus wichtige Einzelthemen bisweilen nicht adäquat adressiert werden können. Dies führte zur Implementierung von bis zu 3 Einzelgesprächen (ggf. zusätzlich auch mit Eltern oder Partner).

\section{Fallbeispiel}

Ein 33-jähriger arbeitsloser Gerüstbauer stellt sich initial selbstständig über die Suchtambulanz mit einem Syndrom süchtigen Verhaltens bei Alkohol- und Cannabisabhängigkeit zum qualifizierten Entzug vor. Nach der qualifizierten Entzugsbehandlung wird Herr B. als Teilnehmer für die AeWA-Gruppe vorgeschlagen, da neben der Suchtkomponente auch impulsives und teils aggressives Verhalten von Bedeutung sind. Im Vorgespräch berichtet Herr B., dass es infolge des Alkoholkonsums zu diversen Straftaten gekommen sei; aktuell sei er zu einer Bewährungsstrafe von 2 Jahren aufgrund von schwerer Körperverletzung verurteilt. In Bezug auf die derzeitige Symptomatik beschreibt er ein hohes Risiko für Impulsdurchbrüche. Seit seinem 13. Lebensjahr bestünden Schwierigkeiten, insbesondere verbale und handgreifliche aggressive Impulse zu kontrollieren. Er gerate oft in Streitigkeiten und Konflikte, auch wegen geringfügiger Angelegenheiten (z.B. Vorfälle im Straßenverkehr). Zudem zeige sich eine emotionale Instabilität in Form von schnellen intensiven Wechseln insbesondere von Wut und Ärger einerseits, andererseits sei er aber auch schnell verletzt und ängstlich. Bezüglich selbstverletzender Verhaltensweisen berichtet er davon, mitunter gegen harte Gegenstände zu treten. Er habe sich hierdurch u. a. eine schwere Beinverletzung zugezogen. Weiterhin habe er sich auch häufiger absichtlich in Prügeleien begeben. Hinsichtlich seiner Biografie berichtet Herr B., dass beide Eltern heroinabhängig gewesen seien und er in der Kindheit viel häusliche Gewalt durch einen nahen Familienangehörigen erlebt habe. Von einer Teilnahme an der Gruppentherapie erhoffe er sich nun, wieder ein normales Leben zu führen und mehr Stabilität zu erreichen. Auf der Grundlage des Vorgesprächs, in dem eigen- und fremdaggressive Verhaltensweisen eruiert werden konnten, sowie weiterer Diagnostik (MINI-DIPS, PID-5-BF, MOAS) wird die Indikation für eine Teilnahme an der AeWA-Gruppe gestellt. Des Weiteren wird der Patient über sein Störungsbild (emotional instabile Persönlichkeitsstörung: impulsiver Typ) aufgeklärt. Der Zusammenhang zwischen vorgefallenen Konflikt- bzw. Deliktsituationen und psychischer Störung sowie der Zusammenhang mit zusätzlichen kriminogenen Faktoren werden erläutert.

Der Patient verfügt über keine hinreichende Fähigkeit zur Emotionsregulation. Er erlebt anlässlich von Differenzen mit verschiedensten Menschen Emotionen wie Ärger und Angst vor negativen Konsequenzen, wenn er diesen deutlich die Meinung sagt. Seine Gedanken rasen, er ist unruhig und angespannt und hat den Impuls, um sich zu schlagen. Als Beruhigung greift er zu Bewältigungsstrategien wie Substanzkonsum, exzessiv Sport zu treiben sowie zur Unterdrückung bzw. Bekämpfung von Emotionen. Nach Abklärung von Indikation und Motivation wird Herrn B. ermöglicht, an den Einführungssitzungen mit anderen potenziellen Programmteilnehmern teilzunehmen. Herr B. zeigt sich im Hinblick auf die Teilnahme an der Gruppentherapie zwar motiviert, ist jedoch teilweise misstrauisch gegenüber anderen Gruppenteilnehmern.

Herr B. erhält einen tiefgehenden Einblick in die Ätiologie seiner Erkrankung. Gruppenregeln werden gemeinsam erarbeitet, z.B. Abmelden bei Nichterscheinen, Akzeptanz der Schweigepflicht, Teilnahme nur nüchtern (ohne Alkohol- oder Drogeneinfluss). Individuelle Therapieziele werden erarbeitet. Die Gruppenbehandlungen basieren auf einer systematischen und manualisierten Abfolge von vier Behandlungsmodulen. In der ersten Therapiephase erlernt Herr B. innere Achtsamkeit, Stresstoleranz, bewussten Umgang mit Gefühlen und zwischenmenschliche Fertigkeiten sowie das Erkennen dysfunktionaler Bewältigungsstrategien (angelehnt an die DBT). Herr B. nutzt im Verlauf Paargespräche und erkennt lebensgeschichtlich geprägte Konflikte und Beziehungserfahrungen. Die Gespräche werden als Entlastung wahrgenommen. Dysfunktionale Verhaltensweisen werden in Form von Verhaltensanalysen im Gruppenkontext nachbesprochen. Herr B. kann das Feedback der anderen Teilnehmer immer besser annehmen, und es gelingt ihm, eigene Sichtweisen zu flexibilisieren. Durch Rollenspiele kann er üben, eigene Gefühle adäquat auszudrücken und Bedürfnisse zu formulieren. Darüber hinaus klärt Herr B. seine berufliche Situation und nimmt Beratungsgespräche bei der Schuldnerberatung auf. Herr B. entscheidet sich für einen neuen Weg und die mitunter noch auftretenden Impulsdurchbrüche haben bei weitem nicht mehr die Ausprägung wie vor Beginn der Gruppentherapie. Es gelingt die Förderung der Motivation, zukünftig ein deliktfreies Leben führen zu wollen und Verantwortung für sich selbst zu übernehmen. In der letzten Therapiephase wird eine Rückfallprophylaxe erarbeitet, und weitere Behandlungsmöglichkeiten (z.B. Einzeltherapie) werden besprochen.

\section{Fazit und Ausblick}

Zusammenfassend lassen sich folgende vorläufige Schlussfolgerungen ziehen: Bis heute gibt es nur unzureichende Behandlungsangebote in Allgemeinpsychiatrien sowie im ambulanten Bereich für Patienten mit gewalttätigen Impulsen bei Persönlichkeitsstörungen. Vor allem psychotherapeutische Behandlungsansätze können hilfreich sein, diese 
Versorgungslücke in der Allgemeinpsychiatrie zu schließen. In der AeWA-Gruppe werden bedarfsadaptiert Strategien vermittelt und Kompetenzen im Umgang mit Aggression entwickelt, welche diesem Patientenkollektiv eine Richtlinien-Psychotherapie in der Folge überhaupt erst ermöglichen können.

Das gruppentherapeutische Konzept wurde im Rahmen des Pilotprojekts von den Patienten gut angenommen. Dies zeigte sich einerseits in einer hohen Teilnehmerkonstanz sowie auch in den Rückmeldungen der Patienten. Als besonders positiv wurde zunächst die Kombination aus Einzel- und Gruppentherapie empfunden. Weiterhin schienen aus Sicht der Patienten das Erkennen und richtige Einordnen der eigenen Emotionen und das Erlernen von Regulationsfähigkeiten, ohne aggressiv zu reagieren, wichtige Elemente der Gruppentherapie zu sein. Von Bedeutung sind v. a. die Indikationsstellung einer Krise und ein schneller Behandlungsbeginn in verschiedenen Settings (Einzelund Gruppentherapie, Paartherapie) mit dem Ziel, präventiv in der Allgemeinpsychiatrie ein Angebot zu schaffen und eine Lücke zu schließen. Durch den frühen Zugang zu umfassender Diagnostik wird eine schnelle Behandlung ermöglicht und dadurch die Gefahr einer Chronifizierung verringert. Durch einen unkomplizierten und strukturierten Zugang zur AeWA-Gruppe sollen unnötige Mehrfachaufnahmen/-untersuchungen verhindert werden, die Dauer der Arbeitsunfähigkeit verkürzt und stationäre Aufenthalte, Rehospitalisationsraten und Frühberentung sowie Straftaten verringert bzw. verhindert werden.

Untersuchungen zur Wirksamkeit des Konzeptes bezüglich der Verringerung von impulsivem Verhalten und Selbststigmatisierung sowie der Steigerung der Lebensqualität sind angelaufen. Weitere Studien sind geplant.

Funding Open Access funding enabled and organized by Projekt DEAL.

Interessenkonflikt R. Schröter, M. Lambert, A. Karow, D. Lüdecke, J. Gallinat und P. Briken geben an, dass kein Interessenkonflikt besteht.

Open Access Dieser Artikel wird unter der Creative Commons Namensnennung 4.0 International Lizenz veröffentlicht, welche die Nutzung, Vervielfältigung, Bearbeitung, Verbreitung und Wiedergabe in jeglichem Medium und Format erlaubt, sofern Sie den/die ursprünglichen Autor(en) und die Quelle ordnungsgemäß nennen, einen Link zur Creative Commons Lizenz beifügen und angeben, ob Änderungen vorgenommen wurden.

Die in diesem Artikel enthaltenen Bilder und sonstiges Drittmaterial unterliegen ebenfalls der genannten Creative Commons Lizenz, sofern sich aus der Abbildungslegende nichts anderes ergibt. Sofern das betreffende Material nicht unter der genannten Creative Commons Lizenz steht und die betreffende Handlung nicht nach gesetzlichen Vorschriften erlaubt ist, ist für die oben aufgeführten Weiterverwendungen des Materials die Einwilligung des jeweiligen Rechteinhabers einzuholen.

Weitere Details zur Lizenz entnehmen Sie bitte der Lizenzinformation auf http://creativecommons.org/licenses/by/4.0/deed.de.

\section{Literatur}

American Psychiatric Association (Hrsg) (1994) Diagnostic and statistical manual of mental disorders, 4. Aufl. American Psychiatric Association, Washington D.C.

Antonowicz DH (2005) Thereasoning and rehabilitation program: outcome evaluations with offenders. In: McMurran M, McGuire J (Hrsg) Social problem solving and offending. John Wiley \& Sons Ltd, Chichester, S 163-181

Aydin N, Fritsch K (2015) Stigma und Stigmatisierung von psychischen Krankheiten. Psychotherapeut 60:245-257. https://doi.org/ 10.1007/s00278-015-0024-9

Azevedo J, Vieira-Coelho M, Castelo-Branco M, Coelho R, Figueiredo-Braga M (2020) Impulsive and premeditated aggression in male offenders with antisocial personality disorder. PLoS ONE 15:e229876. https://doi.org/10.1371/journal.pone.0229876

Bateman A, Fonagy P (2006) Mentalizing and borderline personality disorder. In: Allen JG, Fonagy P (Hrsg) Handbook of mentalization-based treatment. John Wiley \& Sons, Chichester, S 183-200

Bundespsychotherapeutenkammer (2018) Ein Jahr nach der Reform der Psychotherapie-Richtlinie

Burlingame GM, Strauss B, Joyce AS (2012) Change mechanisms and effectiveness of small group treatments. In: Lambert MJ (Hrsg) Bergin \& Gareld's handbook of psychotherapy and behavior change, 6. Aufl. Wiley, New York, S 640-690

Cowman S, Björkdahl A, Clarke E, Gethin G, Maguire J, European Violence in Psychiatry Research Group (2017) A descriptive survey study of violence management and priorities among psychiatric staff in mental health services, across seventeen european countries. BMC Health Serv Res 17:59

Feng CY, Chu H, Chen CH, Chang YS, Chen TH, Chou YH, Chang YC, Chou KR (2012) The effect of cognitive behavioral group therapy for depression: a meta-analysis 2000-2010. Centre for Reviews and Dissemination,

Fiedler P, Herpertz S (2016) Persönlichkeitsstörungen, 7. Aufl. Beltz, Weinheim Basel

Gendreau P, French S, Gionet A (2004) What works (what doesn't work): The principles of effective correctional treatment. J Community Correct 13:4-6

Guy W (1976) Clinical global impressions. In: ECDEU assessment manual for psychopharmacology. National Institute of Mental Health, Rockville, S 217-222

Hautzinger M (2011) Gruppentherapie. In: Linden M, Hautzinger M (Hrsg) Verhaltenstherapiemanual, 7. Aufl. Springer, Berlin Heidelberg, S 393-401

Herpertz S, Mancke F, Bertsch K (2016) Aggressivität, emotionale Instabilität und Impulsivität bei der Borderline-Persönlichkeitsstörung. Forens Psychiatr Psychol Kriminol. https://doi.org/10.1007/ s11757-016-0379-y

Kay SR, Wolkenfeld F, Murrill LM (1988) Profiles of aggression among psychiatric patients: I. Nature and prevalence. J Nerv Ment Dis 176:539-546. https://doi.org/10.1097/00005053-19880900000007

Klauber J, Geraedts M, Friedrich J, Wasem J (Hrsg) (2018) Schwerpunkt: Bedarf und Bedarfsgerechtigkeit. Schattauer, Stuttgart

Krueger RF, Derringer J, Markon KE, Watson D, Skodol AE (2012) Initial construction of a maladaptive personality trait model and inventory for DSM-5. Psychol Med 42:1879-1890. https://doi. org/10.1017/S0033291711002674

Linehan M (1996) Dialektisch-behaviorale Therapie der BorderlinePersönlichkeitsstörung. CIP-Medien, München

Linehan M (2008) Dialektisch-behaviorale Therapie der BorderlinePersönlichkeitsstörung. CIP-Medien, München (Korrig. Aufl)

Mancke F, Herpertz SC, Bertsch K (2018) Correlates of aggression in personality disorders: an update. Curr Psychiatry Rep 20:53. https://doi.org/10.1007/s11920-018-0929-4 
May DD, Grubbs LM (2002) The extent, nature, and precipitating factors of nurse assault among three groups of registered nurses in a regional medical center. J Emerg Nurs 28:11-17. https://doi.org/ $10.1067 /$ men.2002.121835

Nitschke J, Sünkel Z, Mokros A (2020) Die forensische Präventionsambulanz Ansbach: Evaluation des Modellprojekts zur Behandlung psychiatrischer Risikopatienten. Nervenarzt 91:439-445. https://doi.org/10.1007/s00115-019-0752-0

Polnay A, James VAW, Hodges L, Murray GD, Munro C, Lawrie SM (2014) Group therapy for people with bulimia nervosa: Systematic review and meta analysis. Psychol Med 44:2241-2254. https:// doi.org/10.1017/S0033291713002791

Ross RR, Fabiano EA, Ewles CD (1988) Reasoning and rehabilitation. Int J Offender Ther Comp Criminol 32:29-35. https://doi.org/10. 1177/0306624X8803200104

Rubio-Valera M, Luciano JV, Ortiz JM, Salvador-Carulla L, Gracia A, Serrano-Blanco A (2015) Health service use and costs associated with aggressiveness or agitation and containment in adult psychiatric care: a systematic review of the evidence. Bmc Psychiatry 15:35. https://doi.org/10.1186/s12888-015-0417-x

Schandrin A, Norton J, Raffard S, Aouizerate B, Berna F, Brunel L, Chereau-Boudet I, D'Amato T, Denizot H, Dubertret C, Dubreucq J, Faget C, Fond G, Gabayet F, Llorca PM, Mallet J, Misdrahi D, Passerieux C, Rey R, Schurhoff F, Urbach M, Bonnet S, Capdevielle D, Andrianarisoa M, Aouizerate B, Bazin N, Berna F, Blanc O, Brunel L, Bulzacka E, Capdevielle D, Chereau-Boudet I, Chesnoy-Servanin G, Danion JM, D'Amato T, Deloge A, Delorme C, Denizot H, Dorey JM, Dubertret C, Dubreucq J, Faget C, Fluttaz C, Fond G, Fonteneau S, Gabayet F, Giraud-Baro E, Lacelle D, Lançon C, Laouamri H, Leboyer M, Le Gloahec T, Le Strat Y, Llorca PM, Mallet J, Metairie E, Misdra- hi D, Offerlin-Meyer I, Passerieux C, Peri P, Pires S, Portalier C, Ramet L, Rey L, Roman C, Schandrin A, Schürhoff F, Tessier A, Tronche AM, Urbach M, Vaillant F, Vehier A, Vidailhet P, Vilà E, Yazbek H, Zinetti-Bertschy A (2019) A multi-dimensional approach to the relationship between insight and aggressiveness in schizophrenia: findings from the FACE-SZ cohort. Schizophr Res 204:38-45. https://doi.org/10.1016/j.schres.2018.07.029

Schaub VA (2018) Verhaltenstherapeutische Gruppentherapie in der Psychiatrie. Kerbe 3:4-6

Sheehan DV, Lecrubier Y, Sheehan KH, Amorim P, Janavs J, Weiller E, Hergueta T, Baker R, Dunbar GC (1998) The Mini-International Neuropsychiatric Interview (M.I.N.I.): the development and validation of a structured diagnostic psychiatric interview for DSMIV and ICD-10. J Clin Psychiatry 59(Suppl 20):22-57

Sipos V, Schweiger U (2018) Gruppentherapie: ein Handbuch für die ambulante und stationäre verhaltenstherapeutische Praxis, 2. Aufl. Kohlhammer, Stuttgart

Statistisches Bundesamt (2020) Rechtspflege. Strafverfolgung 2019. Destatis, Wiesbaden

Steinert T, Hirsch S (2020) S3-Leitlinie Verhinderung von Zwang: Prävention und Therapie aggressiven Verhaltens bei Erwachsenen. Nervenarzt 91:611-616. https://doi.org/10.1007/s00115019-00801-2

Steinert T, Traub H-J (2016) Gewalt durch psychisch Kranke und gegen psychisch Kranke. Bundesgesundheitsblatt 59:98-104. https://doi.org/10.1007/s00103-015-2262-y

Webster CD, Douglas KS, Eaves D, Hart SD (2017) Assessing risk for violence (Version 2). J Forensic Pract 19(2):130-138. https://doi. org/10.1108/JFP-08-2016-0039

Young JE (1990) Cognitive therapy for personality disorders: A schema-focused approach. Professional Resource Exchange, Sarasota 